only the British Museum Library and the National Libraries of Scotland and Wales are mentioned. more than in passing ; of the university libraries only those of Oxford and Cambridge; and the special libraries are even more sketchily noted. There is no reference to any library maintained by a professional society such as the Chemical Society, and the description of the public library service is likewise extremely condensed, though it is obvious from the comments made that the writers of the report have seen and pondered a great deal they have been unable otherwise to mention. The slenderness of most library staffs is stressed, but in noting the favourable comments on buildings and equipment it should be realized that most of the libraries visited were in new buildings, not always completely finished or equipped.

\section{Japanese Planetarium Equipment}

Messrs. Chiyoda Kogaku Seiko K.K., manufacturers of Minolta cameras, have now developed a series of Minolta planetarium projectors. These projectors are distributed in the United Kingdom by Japanese Cameras, Ltd., Tunstall, Stoke-on-Trent. There are four standard projector models in the series priced in the range $£ 7,000$ - $\$ 69,000$. Models $S$ and III are remote controlled and require a dome the diameter of which is in the range 66-100 ft., while models $M S$ and $M$ are controlled from a position adjacent to the projector and require a dome the diameter of which is in the range 20-50 ft. Models $M S$ and $M$ project about 3,000 stars, and projectors are installed for the movements of the planets. These planetary projectors are quite separate in the case of model $M S$, but are coupled in the case of model $M$. Model $S$ is similar to model $M S$ except that it carries a projector giving 9,000 stars and a separate bright star projector. Model III, the most refined of the series, is similar to model $S$ with regard to star projection, but incorporates the coupling of the planetary projectors as in model $M$ as well as other devices. Each of the four standard projectors carries an equivalent set of special effects projectors, such as a Milky Way projector, a morning- or evening-glow projector, etc. Model III carries a constellation projector in addition. The standard projectors may be supplemented with auxiliary projectors to reproduce auroral phenomena, the solar system as viewed by an external observer, etc. It is interesting to observe that an auxiliary projector can be provided to illustrate the motion of artificial satellites-a choice of American or Russian being provided. It is to be hoped that the advent of cheaper projectors may encourage the establishment of more planetaria in Britain. Small instruments, not necessarily operating on a commercial basis, could be used to assist the teaching of astronomy in schools and colleges, especially if situated in large centres of population.

\section{Medicine, Science and the Law}

THReE papers, which appear in Medicine, Science and the Law (1, No. 4; July 1961), contain implications of considerable importance; each is a real advance in the development and refinement of scientific methods of identifying blood. Dr. R. A. A. Coombs and Barbara Dodd describe a microscopical technique which enables them to determine the nature of a stain on a single fibre of clothing or other material. They show that it is now possible to say with a high degree of certainty not only that the fibre is stained with human blood but also to determine, at loast within the limits of the $A B H$ system, the group to which the blood belongs. The method is applicable to stains of considerable age, certainly up to three years, in the case of human blood. Dr. H. H. Muller and his colleagues have examined the problem of whether stains consist of human or animal blood in a different way. They have evolved a mothod of testing for hæmoglobin in the stain. Hæmoglobin differs in its minute physical and chemical characteristies from species to species, but it has hitherto been difficult to identify the species from which it came. The method suggested does not give information about the group of the blood in question. Dr. I. Dunsford, R. Lodge and W. Spielmann describe an investigation into blood groups in a paternity case in which, after a detailed examination of the groups of the child and putative father using all the refine. ments in technique normally available, the blood groups of the putative father fitted the blood groups of the child in every aspect but one. The child's $R h$ constitution was shown to be $C D E / c d e$ and the putative father's more probably $C D e / c D E$ but possibly $C D E / c d e$ (that is, as in the child). The technique described in their paper enabled the authors to show that the putative father's $R h$ constitution was $C D E / c d e$, so that he was not excluded. Moreover, the 'fit' of his blood with the child's is so good that (although the authors do not claim it) the scientific evidence points to his being in fact the father.

\section{Blindness in Africans in Northern Rhodesia}

IN 1949 territorial ophthalmic services were started in Northern Rhodesia. Before that date very little was known of the incidence of eye diseases and even less about the extent and causes of blindness. In 1950 a simple blindness register card was produced and distributed to all administrative officers, together with a simple memorandum on eye diseases describing the four conditions which are the principal causes of blindness. Dr. C. M. Phillips, Government ophthalm. ologist, Lusaka, now shows (Central African J. of Med., 7, No. 5; May 1961) that the overall incidence of blindness is considered to be 500-750 per 100,000 ; in some areas it rises as high as 2,300 , with the unique feature of having a higher incidence of onset in childhood than in adulthood. The main causal lesions of 2,701 patients examined are corneal pathology, phthisis bulbi, senile cataract and trachoma. It is considered that the predominant factor responsible for the first two lesions is traditional African medicine. Taking all factors into consideration, it is believed that four out of every five persons who have lost their sight did so from a cause that is either preventable or curable.

\section{Uses of Castorseed}

A Two-DAy seminar on Castorseed and its Products was held during August 11-12, 1960, at the Regional Research Laboratory, Hyderabad, under the sponsorship of the Council of Scientific and Industrial Research, India. In order to consider the various factors bearing on the present and future of the castor industry in India, a number of questions likely to arise were first formulated under suitable heads. These were sent to a large number of research institutions, organizations dealing with castor, and firms either possessing castor or utilizing its products, especially castor oil. Data or information illustrating and amplifying the questions raised were requested. The data, received in the form of brief notes, were 